\title{
On Avoiding Research into the Blindingly Obvious
}

\author{
Peter A Aspinall \\ Professor Emeritus, School of Built Environment, Heriot Watt University, Edinburgh UK.
}

doi:10.5618/arch.2012.v1.n1.8 || Received: 26-03-2012, Accepted: 16-05-2012, Available online:21-05-2012

\begin{abstract}
Empirical research in certain areas of environmental design and behavioural studies is faced with a credibility dilemma. This is because its area of investigation overlaps with public and professional experience but does not formally or adequately acknowledge this experience in its methodology. As a consequence many research outcomes while evidence based can often be blindingly obvious. An attempt is made here to provide a philosophical framework to avoid this dilemma. This is the empirical constructivist position of Bas van Fraassen coupled with a Bayesian approach to hypothesis testing. It is argued that this framework has theoretical and pragmatic benefits for examining inter relationships between outcomes from quantitative and qualitative methodologies.
\end{abstract}

Keywords: research; theory; Bas van Fraassen; Bayes; environmental design

\section{Introduction}

This paper is about the nature of research in environmental design related disciplines such as urban design and landscape architecture. It is not directed at design based practice research (although it might well have wider relevance there) but rather at the role of science or evidence based research in such practices. The outcomes from such research raise a dilemma which to a greater or lesser degree many of us may face.

A simple and useful definition of research is that it is a process which provides new knowledge or insights. This general definition can be seen as having two consequences. Firstly research findings should either have the capacity to inform or surprise a reader (particularly those working in the relevant field) or the findings should help reduce uncertainty around a controversial topic. If surprise is considered too strong then before a study is carried out there should at the very least be an element of unpredictability about its conclusions. It is also worth recollecting Poppers view [1]. He argued that many statements can be true but can carry very little informational content e.g bland statements such as 'it will rain'. The extreme case is a necessary truth i.e a tautology which carries no information at all. It is reasonable to assume therefore that the informational content in a research conclusion is in inverse relationship to its likelihood of being made. With this in mind consider the following which are representative of some conclusions from funded research findings in the general environmental area.

A research project on commuter attitudes to transport concludes with a statement that travellers are most annoyed if trains do not run on time, and not running on time will lead to increased passenger stress; or a project in urban design concludes that older people need benches (and toilets) at regular intervals in urban areas if they are to be encouraged to get out and about; or the 'finding' that unpleasant and noisy neighbourhood environments will discourage people from going out for a walk.

It is difficult to imagine anyone (whether general reader or specialist) being surprised or unable to anticipate any of these 'findings'. It's also difficult to imagine what expectations the funders had when funding the projects (if other than the blindingly obvious) let alone what possible theoretical or controversial issue has been advanced or resolved. It's not clear therefore what this research based empirical evidence is doing other than opening research in the field to public and professional ridicule.

On the other hand it is important to recognise that the type of research investigation in question does have the potential to inform design. Questioning common sense is a good thing but usually in circumstances where there is a likelihood of difference or uncertainty in opinion. For example an important strand of urban design is about how environments might meet peoples needs, and facilitate greater levels of activity and social contact with consequent health benefits. Environmental design solutions to address quality of life, satisfaction or health are by no means obvious. And again theoretical concepts such as environmental affordance [2] which are of interest in environmental psychology are of undoubted relevance to designers. On the other hand for many general design related behavioural studies no empirical research is needed at all. For these situations 
there are a sufficient number of designers with the appropriate knowledge base and experience to produce good creative solutions and it is an insult to designers to assume otherwise.

One underlying issue here is how we come to know things. There is a longstanding and uneasy alliance between qualitative and quantitative research. At a psychological (if not a methodological) level there appears to be a dichotomy and lack of trust between the two sets of protagonists over the validity of their findings. What's more, the two methodologies don't appear to be seen as two alternative and legitimate windows onto the same underlying reality. What is needed therefore is:

a) a way of identifying those empirical consequences of design in need of answers i.e. separating consensus and 'obvious' professional views from debatable or controversial views, and

b) a move to direct qualitative and quantitative research at the latter.

Perhaps of greater concern is that lurking behind the funding and general political drive towards evidenced based policy (and hence amorphous empirical research) might be outmoded assumptions about environmental determinism. This bedevilled early research in environmental psychology and architectural studies and generated misguidedly optimistic views about what environmental psychology might offer designers [3]. We also need to remember that while environmental design can have a significant effect on behaviour and wellbeing, the effect in most cases is relatively small in relation to many other factors - e.g. particularly social ones [4]

From a different perspective, the inter-relationship between the quantitative objective and rational and the qualitative subjective and emotional has been the subject of recent research in neuroscience [5]. This has challenged lingering assumptions about the separation of reason and emotion, and the role of left and right hemisphere activity in our thinking. It appears that even in 'rational decisional situations' the left rational hemisphere is far from being the dominant partner. On the contrary the right emotional hemisphere would appear to be central to general holistic assessments of what is going on and to the consequent selection or filtering of 'relevant' incoming data. The lefts specialty is in language and analysis. However once any analysis is complete it is back to the right hemisphere to take the wider vision for decision or action. McGilchrist [6] has argued that the European emphasis on precise categorical thinking since Plato is distorting our ideas about science, and our notions of objectivity. In particular the dualistic splits where for example nature is seen as a detached object separate from us, reinforces this false notion of the objective and leads to unhelpful distinctions about quality and quantity.

Given the uneasy alliance between the qualitative and quantitative the question arises as to whether there is a theoretical framework which helps reconcile these major methodological differences? And at a detailed level will this framework help produce empirical research which meets appropriate criteria and yet avoids overlapping with the blindingly obvious.

\section{Aim}

What follows is both theoretical and practical. The conceptual framework to be proposed is a mix of ideas from the philosophy of science - in particular those by Bas van Fraassen [7] and Thomas Bayes. (early $18^{\text {th }}$ century non conformist minister - see ( 8) ). It is hoped to show that the qualitative and quantitative while different in their approach, overlap to a greater degree than is often realised at the inferential and decisional level, and in the believability and degree of acceptance of their conclusions. Three approaches will be made in an attempt to demonstrate this:

a) a view of science will be presented which is less dogmatic and rigid than that commonly assumed to hold. By relaxing misconceived constraints around quantitative measurement it might appear more accessible and relevant to design.

b) an attempt will be made to strengthen the role and robustness of the qualitative through links to the quantitative. For example there are formal methods within psychology which can help clarify to ourselves and others our subjective beliefs in uncertain situations. [9]

c) the notion of Bayesian degrees of belief or acceptance will be introduced as a possible unifying theme behind the two methodologies.

\section{Science and reality - the antirealist view of science.}

The intention in this section is to move towards the antirealist views of science proposed by Bas van Fraassen [7]. However before presenting these views some prior philosophical context, however sketchy, is needed. If we take a realist view of science we believe there is a world out there to be investigated and that science tells us in a literal way about that world through its theories and its findings. For example, if science tells us there are new invisible fundamental particles, then even if we cant see them we believe they are there.. However this realist position is an oversimplification of the situation. There 
has been a longstanding philosophical debate regarding secondary qualities. [10]. For example a widespread assumption is that the colours we see are properties of external objects determined by the environmental context of their surface and the prevailing light. But the colours we see are of course also dependent on the properties of our sensory system. They are anthropocentric. Seeing colours involves the disposition of an object to activate certain neuronal structures of our brain. The structures are just as important as the external surfaces in determining the perceived colours. It would therefore be incorrect to say colours are externally determined. It would also be incorrect to say they are the product of our sensations. Both are needed in an interdependent relationship - an early forerunner to the biological parallel of environmental affordances introduced by J.J.Gibson [11] and now discussed in a landscape context [2].

However well before landscape interest in affordances Kant [12] had made a similar but more radical and startling claim. It is that we can never know the world as it is because we have no direct contact with it. Everything we know is mediated by our mind and its cognitive structures. Kant made an important distinction between the noumena (i.e. properties of the world independent of us) and the phenomena (our knowable experience of the world). So the empirical world which science investigates is not (even in the investigation of matter) independent of us. As a methodology, science comes closest to giving us access to the noumena but our cognitive processes are always at work in our theories and mind generated constructions for interpreting data.

More recently Thomas Kuhn [13] in agreeing with Kant also believed that the world which scientific theories depict is not independent of the intellectual activities of the scientists - it is a phenomenal world. Furthermore scientific revolutions are times when the world doesn't change but the human contribution to the world changes. Kuhn describes scientific paradigms in which scientists (and professionals) hold sets of assumptions about their working world to get on with day to day life (including doing research). When a new finding occurs which contradicts previous findings it needs accommodating, which leads to a paradigm shift. Now lots of our former beliefs are dropped and new ones take their place. At any point in time there are the two elements - the evidence itself and our constructions about the evidence. For Kuhn there were multiple phenomenal worlds such as that before and after a scientific revolution. He considered this change often so dramatic that theories on either side of the change are in Kuhn's terms 'incommensurable' such that the conceptual structures of one are not simply in conflict but deny other alternatives. In this situation theories are dividing the world up in different ways and are talking about quite different things. The Kuhnian analysis implies that there is a common noumenal world underpinning different phenomenal accounts. The noumenal aspect might on occasions be proportionately small but it's there. Apparently incompatible accounts might therefore both be correct because they are descriptive of different worlds. Therefore we can retain conflicting content because it doesn't imply the world itself is in some self contradictory state.

\section{The immersive solution}

It is a development of these ideas that has been presented by Bas van Fraassen [7] in a seminal and controversial book 'The scientific Image'. Following Kant and Kuhn he argues for an antirealist position which he describes as 'constructive empiricism'. His philosophical position has three components.

The first component is semantic. Scientific theories are to be given a literal meaning and understood as a realist would understand them. They are not metaphors or phenomenological representations, they are descriptions of a possible noumenal world.

The second methodological component is immersion. To immerse oneself in a theory is not to believe that it is true but to imaginatively enter into the world of the theory, accepting it as if it were true and working within it. So for example if I was to carry out an experiment on the diffraction of light I would accept the wave theory of light rather than the particle theory of light to which I might switch acceptance for studying the refraction of light. The third component is epistemic and acknowledges the fact that scientists frequently don't actually believe their best theories. According to van Fraassen scientists should be agnostic towards any theory but at the same time be able to consider it empirically adequate. When two theories are in conflict (as in the wave or particle view of light) my agnosticism allows me to avoid the conflict by focussing on the describable empirical outcomes. This means that in accepting a theory we need believe only part of it - that part which we empirically observe or which might have occurred from the theory. This is the distinction between the antirealist view and the realists who share belief in both the literal semantics and in addition the wider content of the theory.

Within this third epistemic component of acceptance, things become more complicated. This is the area of partial belief in which we believe some but not all the consequences of a theory and remain agnostic about the 
rest. There seem to be useful parallels here in the situation of a design studio where it seems crucial for learning that a student be immersed in and accept the form of life of the studio - in other words to use its language and follow its principles - even though the student may be skeptical about their credibility or value. Acceptance doesn't mean belief. Remember that according to Bas van Fraassen the parts we should believe are the empirical confirmations of claims about observable states. There is a way of expressing our uncertainties and partial beliefs or degrees of acceptance through the work on probability carried out by Thomas Bayes 300 years ago. However before arriving at Bayes there are one or two remarks needed on conventional statistics.

\section{Conventional statistics}

The criteria for significance in conventional statistics have been established by the statistical community and it would be foolish to question these criteria. Nonetheless the purpose of the criteria was to minimise the possibility of making errors concerning evidence based inferences. It would seem sensible therefore to look at some pragmatic consequences of the criteria for those involved in research in environmental design.

a) The testing of a hypothesis is open to two kinds of error. The first is stating there is a significant finding when there is not (called alpha). The second is saying there is not a significant finding when there is (called beta). In science (which errs on the conservative) alpha is considered a more serious error to make than beta. That's why the likelihood of alpha $(\mathrm{p}=0.05)$ is kept much lower than beta which on statistical power grounds is typically set at $\mathrm{p}=0.2$. (Power $=1$-beta). The important point is that for any particular empirical set of data, the sum of alpha and beta is constant. So if we make alpha bigger we automatically make beta smaller and vice versa. Now if we move from statistical testing to real world decision making situations we face exactly the same problem - i.e. there are two ways of being wrong. We can say an action is appropriate when it isn't and say it isn't when it is. Suppose a drug is introduced as a vaccine against swine flu. Assuming swine flu is a mild ailment we want a drug which is highly effective at curing swine flu (i.e. very significant) with an extremely low chance of side effects - for example a value much less than $p=0.01$. On the other hand if someone is seriously ill and other medications have already been unsuccessful, then we might try out a drug with a lower chance of success (even one which has not reached significance (i.e. $p>0.05$ ) and which may have a significant chance of side effects.
In other words it is the context and the consequences of our actions which determine how we act and follow through decision making on probabilistic evidence. It is worth recalling that when R.A.Fisher [14] recommended a $p$ value at 0.05 it was in response to a request to find the number of trials necessary to confirm a claim a woman had made that she could detect whether milk had been placed first or second in a cup of tea. At the other extreme the physicists working on the Hadron Collider are using a value of $p<0.0001$ to establish the existence of Higgs Boson.

b) In determining the statistical criteria for significance or non-significance statisticians have agreed to set alpha at $(\mathrm{p}=0.05$ or $\mathrm{p}=0.01)$. However a lot of the environmental data gathered in the environmental design related field (say the impact of greenspace on quality of life or health) hovers around one of these thresholds of significance $(\mathrm{p}=0.05)$. A strict application of statistical criteria shows one study just significant at $\mathrm{p}=0.05$; the next just non significant at $\mathrm{p}=0.05$. In this situation there are two ways forward. One way might be to follow Scots law and introduce a middle $\mathrm{p}$ value category of 'not proven'. In other words we would harden the boundaries for significance or non significance and introduce not proven in between. In the context in which we are working this would help stabilise research conclusions. We might then have:

\section{if $\mathrm{p}<0.01$ then significant}

if $p$ between 0.01 and 0.1 then not proven

if $p>0.1$ then not significant

An alternative approach to stabilise findings would be to carry out a meta-analysis across several studies. However within landscape or urban design this research opportunity would seem unlikely mainly because of the small number of replicated studies and the varied situations within which data is gathered. Nonetheless if either of these alternatives had been implemented the context for discussion about the impact of view on recovery from surgery [15] would have benefitted.

c) There is another point which is particularly relevant to applied research. It is often mistakenly assumed that study outcomes with more significant $p$ values have stronger effects than those with less significant $p$ values. For example in the medical field clinicians often assume a result with a $\mathrm{p}=0.001$ has a much greater treatment effect than one where $p=0.03$. This is true but only when sample sizes are the same. It is not true if the lower $\mathrm{p}$ value is associated with a larger sample size. To put this another way, it is possible with a large sample to have a highly 
significant $\mathrm{p}$ value between groups yet a minimum impact of treatment (i.e. small effect size). Statistical significance is not sufficient in itself - results also need to be clinically meaningful. This same issue occurs in regression and correlation. Even with a sample size around $n=140$, a significant correlation at $\mathrm{p}=0.01$ occurs when the correlation between two variables is only as high as $r=0.2$. This actually means that the two variables share $4 \%$ of what they are measuring in common. (shared variance is $r$ squared). It also means that $96 \%$ of what they measure is unaccounted for. In terms of any practical consequences a significant finding at this level is almost useless. Statistical results need to be contextualised.

Several articles have appeared in recent years questioning an over reliance on $p$ values and calling in particular for attention to effect sizes and confidence intervals as moderators of $p$ values [16] [17]. A recent very interesting and radical departure has been to produce regression software which doesn't explicitly include $\mathrm{p}$ values at all, yet which gives better out of sample predictions [18].

*Foot Note: For those unfamiliar with statistical power calculations the 4 inter-related issues are alpha, power (1-beta), effect size, and $n$ the number of cases. Knowing any 3 enables us to determine the $4^{\text {th }}$.

The intention here has been to outline ways in which statistics can become more appropriately tuned to environmental data and the kind of inferences and interventions which environmental designers want to make. It is argued that broadening the context for inference away from specific $p$ values will help stabilise research findings.

\section{Bayesian theory}

The third epistemic component of Bas van Fraassen's philosophical position is partial belief, which takes us to Thomas Bayes. Bayesian ideas on probability have been controversial and marginal within statistics until relatively recently. While still controversial in its definition of probability, increased interest in the Bayesian approach has occurred for two different reasons. Firstly a Bayesian inference can apply to an individual case rather than sample statistics. This has been of particular interest in medicine which (like design) involves an individual case i.e the diagnosis of a particular patient. Secondly with the arrival of fast computers statistical parameters can be empirically generated by running thousands of random samples rather than relying on theoretical definitions of the parameters. (An example is the recent addition of the
Monte Carlo Markov Chain Hierarchical Bayesian algorithm in the AMOS module of SPSS). However the intention here is to introduce philosophical ideas behind Bayesian inference which lead to notions of partial belief and to clarify controversial and non-controversial applications. It should be emphasised at the start that the use of conventional probabilities (i.e.frequencies of events) within Bayesian formulas is a non controversial way of deriving conditional probabilities.

In introducing the ideas there are some key elements.

a) Bayes suggested that instead of probability being defined in terms of the frequency of occurrence of an event, it should be defined as the degree of belief in the likelihood of an event. Numerically, zero would indicate disbelief, 1.0 would indicate complete belief, and values in between would indicate degrees of partial belief - we might think of 0.5 as genuine agnosticism, an equal likelihood of believing or not believing. This is obviously controversial. Is it acceptable to quantify subjective degrees of belief in this way and what's more can we combine them using mathematical relationships?

b) When considering the relationship between evidence and belief (particularly in the situation of theory or hypothesis testing) conventional thinking is turned on its head. In conventional statistics we consider the probability of a hypothesis $\mathrm{p}(\mathrm{H})$ being true given the evidence (e) - written as $\mathrm{p}(\mathrm{H} / \mathrm{e})$. $(\mathrm{H})$ is typically $(\mathrm{Ho})$ or the hypothesis of no difference which we seek to overturn. In a Bayesian world we consider the probability of the evidence (e) given the hypothesis (H) - written as $\mathrm{p}(\mathrm{e} / \mathrm{H})$. This is the inverse of the standard approach.

c) In carrying out any research study we usually begin by reading the literature. This helps formulate and refine our research questions. However when we apply a significance test to our findings in a non Bayesian setting we are confirming or falsifying a null hypothesis as applied to our particular study. The significance test we apply doesn't depend in any way on what others have found in carrying out a similar study. In fact this isolation or independence is seen as the mark of science - a typical neutral stance towards this particular set of data. However Bayesians believe that in arriving at our conclusion we should take on board 'a priori' probabilities which reflect an informed belief about what has gone before. Is our proposed hypothesis highly likely or unlikely to be held or overturned? What is believable before we carry out our study? The apriori probabilities which reflect this wider thinking might come from different sources (e.g - a previous empirical study or a qualitative source,). The findings from our study are 
then mathematically combined with the apriori probabilities to produce the likelihood of different possible conclusions (or likely beliefs) from placing our study in this wider context. If another researcher follows us in carrying out a similar study then the apriori probability for that researcher is not the value we used but the modified value following our research. Our a posteriori probability becomes the new researchers a priori probability, and so on.

d) One interesting feature of the Bayes approach is 'convergence'. Let us suppose that there is an effect which replicated studies might detect but where the initial apriori probability estimate is wildly inaccurate. This will result in a very inaccurate initial outcome a posteriori probability. However although inaccurate the a posteriori probability will be closer to the true value than the initial apriori estimate. With a surprisingly small number of replicated studies each taking the latest a posteriori value as its input apriori value, the outcome probabilities rapidly converge on an estimate which becomes the best approximation available.

e) Putting the situation more formally: for prior belief $\mathrm{Hp}$, the evidence $\mathrm{E}$ can either

i) be neutral to the current view Hp (i.e. prior belief Hp remains the same with or without E) - we might say the evidence is irrelevant to the degree of belief in $\mathrm{Hp}$;

ii) reinforce the current view Hp (i.e. prior belief $\mathrm{Hp}$ is increased in probability and therefore degree of belief); or

iii) go against the current view Hp (i.e. prior belief $\mathrm{Hp}$ is reduced in probability- our degree of belief is less strong).

The advantage of this approach is that research findings are automatically in context. What is considered research could either be findings which reinforce the status quo when this belief is tentatively held, or it could be a shift of belief between possible alternative opinions. This is in contrast to criticisms of a naïve Popperian approach in science - scientists famously did not ditch hypotheses on the grounds of single experiments which falsified them but sensibly waited for evidence to accumulate from several sources [19]. Such scientific practice is in line with a Bayesian approach. Any research project is carried out against a background of similar research. This is, of course, reflected in the literature survey researchers are obliged to write, however in a non Bayesian world it is not reflected in conventional analytical procedures.

Lets turn now to the non-controversial and controversial uses. If probabilities are conventionally understood as frequencies of events then as stated a Bayesian application is non-controversial - although often counter intuitive. For example suppose in a city there are two colours of taxis, $85 \%$ are blue and $15 \%$ are green. An eye witness reporting a hit and run incident says the taxi involved was green. Trials are carried out under lighting conditions similar to those at the accident and the eye witness correctly identifies $80 \%$ of green taxis as green. What credibility do we give to the eyewitness report? Most people will conclude from this that if the person correctly identifies $80 \%$ of green taxis under lighting conditions prevailing at the time of the accident then the likelihood is the taxi involved in the hit and run was green. However this isn't the logical conclusion we should draw.

Lets assume we have no eyewitness evidence at all and that the taxis are the same except for body paint and are randomly distributed across the city. In the absence of any evidence the most likely taxi involved in the accident will be a blue one ( $85 \%$ of the time).This baseline (apriori) probability has now to be modified by the available evidence. An application of the Bayesian formula shows that after inclusion of the evidence the likelihood (p value) the taxi was blue is still greater than the likelihood it was green. The error in the eye witness account although small makes this evidence not sufficient to overturn the apriori bias towards a blue taxi.

Many similar applications of the use of screening tests in medicine have shown counter intuitive conclusions to doctors - particularly that failing a screening test (with a low error rate) does not mean the person has a particular pathology (with low prevalence).

The above examples are not controversial because the conclusions result from a Bayesian application of evidence based conditional probabilities. These conclusions represent the best combination of uncertain evidence in indicating the likelihood of different events or outcomes. However when the application is used in situations where some or all the probabilities are subjective then we are on very controversial ground. However the point I wish to make is that the structure which Bayes brings to this subjective data is more beneficial than its absence. Suppose we try to combine some basic subjective beliefs in the Bayesian framework firstly to reinforce the dilemma over the blindingly obvious and secondly to explore some practical examples.

\section{Blindingly obvious consequences}

The Bayesian view helps us to formally understand the examples in the introduction. . For example imagine 
that for most professionals in urban design the belief that older people needed benches at regular intervals in urban areas would be held with almost complete certainty. As a design option benches would be installed (unless other practicalities prevented it) with almost automatic regularity and little thought because its an obvious thing to do. From a Bayesian perspective this is a situation where the apriori probability (the conventional degree of belief in the value of benches) is already extremely high (say beyond $\mathrm{p}=0.8$ ) before anyone carries out any research. As a consequence, while the empirical findings might shift this probability either way (e.g. say a likely increase to an a posteriori probability of $\mathrm{p}=0.9$ or to a less likely decrease to a probability of $\mathrm{p}=0.7$ ) the outcome of the research is still the same because the $\mathrm{p}$ value is still well above $\mathrm{p}=0.5$ ). Of course this situation would change and become interesting if we ever had a string of empirical studies in which one after the other went against conventional wisdom. Then each would reduce the a posteriori probability so that it began to approach and go below 0.5 as evidence came in. In the case of benches the possibility of serial empirical evidence ever overturning the obvious is almost inconceivable. Research here is worthless. It surely makes more sense to be running empirical studies in situations where conventional professional wisdom is either much lower and therefore where there is greater uncertainty, or in situations where there is genuine controversy.

A good example of the latter is the claim that natural environments bring health benefits such as reduced stress - probably assumed by landscape architects and the general public and evidenced by a few studies from one main source [15]; yet not believed by many scientists in medical research. This is where we need research! See worked examples in appendix.

It should be emphasised that when subjective probabilities are used within the Bayesian framework, the Bayesian version is not necessarily an accurate descriptive account of how people reason. For example experimental evidence shows that people are not naïve Bayesians and that prior probabilities are often not taken into account (although they should be) in the inferential process. [20] [21]. Nonetheless what is presented here is a normative view of how inferences might be made. Bayes offers a structure for partial belief by combining evidence and belief in a more flexible and less prescriptive way than conventional criteria. It also helps ground these beliefs with increased sensitivity to context and previous studies. The purpose of this section is to suggest Bayesian ideas as a practical and theoretical framework for research in complex areas of uncertainty which depend on skilled intuitive judgments. In these circumstances a Bayesian approach is a way of building an inferential knowledge base on a mix of evidence and subjective beliefs. Bayesian ideas may be a constructive way forward for interpreting research findings and for research development., The suggestion is not to return to prescriptive and mechanistic formulaic decisional models. Rather the role of the models is to bring personal insight by clarifying the relationship between different beliefs within the decisional process.

\section{Concluding observations}

The point of view presented here is that in the field of environmental design unnecessary empirical research is being funded and undertaken. The questionable value of this research is seen in its obvious conclusions. There are different reasons for this. One factor seems a lack of focus in the research questions and anticipated outcomes in drives for evidenced based policies; a second is the lack of integration and apparent tension between qualitative and quantitative research methods and conclusions. It is acknowledged that parallel and complementary qualitative and quantitative approaches are used by many research groups including our own. However ideas of integration even if desirable would seem unachievable.

In this paper an attempt is made to put the empirical side of environmental design research in a different theoretical context which is more useful to the relevant professionals and funding agendas. This context is a mix of anti-realist views of science (Bas van Fraassen) and attitudes to uncertainty and partial belief from the work of Thomas Bayes. It is argued that this offers the possibility of a more integrated treatment of qualitative and quantitative research than is currently the case. Indeed greater convergence between the two could be achieved if notions of partial belief were taken more seriously and applied in both research and design situations.

The views outlined would inform and clarify research findings, and avoid research into the blindingly obvious. In addition there is the possibility that these ideas could help students understand why the learning process takes the form it does as they progress through the necessary idiosyncrasies of design studios. Some of my scientific colleagues might think this is a recipe for chaos. My response would be that this is a pragmatic and transparent approach to bring about the possibility of both structure and integration (but not prescription) to this area of environmental design research.

In summary the present proposal is: 
a) to consider the implications for research and environmental design from the Bas van Fraassen approach of 'constructive empiricism'

b) To explore professional opinion (through Bayesian or alternative means) and only carry out empirical research in situations where there is evidence of professional opinion being polarised or uncertain.

c) To produce guidelines on how statistical significance levels might map onto design related consequences and actions

d) To explore whether the Bayesian approach helps structure alternative theoretical views in the discipline.

\section{References}

[1] Popper K (1959) The logic of scientific discovery. Routledge

[2] Heft H (2010) Affordances in the landscape. In Innovative approaches to researching landscape and health. Routledge Abingdon

[3] Stokols D (1987) Handbook of environmental psychology. New York: Wiley

[4] Bonnes M (1995) Environmental Psychology: a psycho-social introduction. Publ. Sage, London

[5] Lehrer J (2009) The decisive moment: How the brain makes up its mind. Canongate books Edinburgh

[6] McGilchrist I (2010) The master and his emissary. Yale books

[7] Bas C Van Fraassen B (1980) The scientific image. Clarendon paperbacks

[8] Sivia D (1996) Bayes data analysis : a Bayesian tutorial. Oxford Univ press

[9] Plous S (1993) The psychology of judgement and decision making. McGraw Hill New York

[10] Locke J (1690) Essays concerning human understanding

[11] Gibson J.J. (1966) The senses considered as perceptual systems. Boston Haughton Mifflin.

[12] Kant (2003) Critique of pure reason Publ. Palgrave Macmillan

[13] Kunn (1962) The structure of scientific revolutions. International encyclopedia of unified science. Univ Chicago press.

[14] Fisher RA. (1925) Statistical methods for research workers, 1st ed. Edinburgh: Oliver and Boyd, Reprinted by Oxford University Press.
[15] Ulrich R (1984) View through a window may influence recovery from surgery. Science Vol 224, p 420

[16] Hoenig J, Heisey D (2001) The abuse of power: the pervasive fallacy of power calculations for data analysis The American Statisticia, Vol. 55, No. 1

[17] Kain Z (2005) The legend of the $p$ value. Anesthesia \& Analgesia November vol. 101 no. 5

[18] Magidson, J. (2010) 'Correlated Component Regression: A Prediction/Classification Methodology for Possibly Many Features', Proceedings of the American Statistical Association.

[19] Harre R(1986) The social construction of emotions. Blackwell Oxford

[20] Cave S (1998) Applying psychology to the environment. Publ L Erlbaum

[21] Kahneman D, Slovic P and Tversky A (1982) Judgements under uncertainty: heuristics and biases. New York. Camb. Univ. press.

[22] Kirk S and Spreckelmeyer K (1988) Creative design decisions: a systematic approach to problem solving in architecture. Publ. Van Nostrand Reinhold

\section{Appendix}

Some worked examples:

Landscape applications. Let us suppose I am interested in Ulrich's [15] claims about the beneficial effects of viewing landscape scenes when recovering in hospital. Positive findings have been published but problems have been encountered over their replication, and we might suppose that scientists are divided over Ulrich's and colleagues' claims. Let us consider the problem from a Bayesian perspective.

\section{a) Bayesian significance without conventional significance}

Initially I estimate the priors. If I am genuinely agnostic or uncertain about the claim (and let us suppose the literature doesn't support one view over another), I can set the two priors $(p(H I)$ and $p(-H 1))$ to 0.5 for the two-alternative case. The best way to see how the probabilities are combined is to set them out in Table 1. The left column represents my main research question and its alternative.

In other words,

a) $p(H 1 / E 1)$ what is the probability of $H I$ being true given evidence $E 1$ (i.e. the probability that landscape scenes do reduce stress, given the evidence in the Ulrich publication) 
b) $p(-H 1 / E 1)$ what is the probability of $\mathrm{Hl}$ being false given evidence $E 1$ (i.e. the probability that landscape scenes do not reduce stress, given the evidence in the Ulrich's publication).

Table 1 Elements for Bayesian Inference

\begin{tabular}{|l|l|l|l|}
\hline $\begin{array}{l}\text { Research } \\
\text { hypothesis, } \\
\text { question or } \\
\text { belief }\end{array}$ & Priors & Evidence & $\begin{array}{l}\text { Probability } \\
\text { estimates for } \\
\text { evidence }\end{array}$ \\
\hline a) $p(H 1 / E 1)$ & 0.5 & $p(E 1 / H 1)$ & 0.9 \\
\hline b) $p(-H 1 / E 1)$ & 0.5 & $p(E 1 /-H 1)$ & 0.3 \\
\hline
\end{tabular}

The second column gives my priors set at 0.5 .

The third column gives

a) $p(E 1 / H 1)$ - what is the probability of getting evidence $E 1$ given $H 1$ is true (i.e. that there is evidence of reduced stress given the Ulrich hypothesis is true

b) $p(E 1 /-H 1)-$ what is the probability of getting evidence $E l$ given $H 1$ is false (i.e. that there is evidence of reduced stress given the Ulrich hypothesis is false)

The fourth column provides illustrative values for the third column and reflects the likelihood of the evidence $\mathrm{E} 1$ given $\mathrm{H} 1$ was true, and below it if $\mathrm{H} 1$ was false. Let us assume these values (from whatever source) are 0.9 and 0.3. This assumption might be based on the subjective judgment of one person, or on the collective judgment of several professionals, or on the outcome of a number of experimental studies.

From Bayes formula

$p(H 1 / E 1)=(0.9 \times 0.5) /((0.9 \times 0.5)+(0.3 \times 0.5))=$ 0.75 , and it follows that $p(-H 1 / E 1)=0.25$.

In other words, given my agnosticism and the evidence, the data has not enabled $p(H / E)$ to reach conventional significance at 0.95 . Nonetheless our belief in the outcome is 0.75 in favour of $p(H / E)$ rather than 0.25 against and so we accept the claim of Ulrich against the alternative.
Furthermore, if someone else carries out research in this area the new priors become 0.75 and 0.25 . In other words we are moving away from total agnosticism of 0.5 and 0.5 towards moderate support for the Ulrich claim. Of course if we wish we can set up intermediate categories from moderate acceptance to conviction where the latter coincides with conventional statistical criteria. Nonetheless the moderate acceptance category is a better logical combination of evidence and subjective belief than $p=0.05$ or nothing!

If the generation of priors appears too uncertain in the brief above account, it is important to note that there are decisional techniques for the generation of subjective probabilities [9]

In the statistical section I suggested a modification to conventional inference with the introduction of "not proven'. Here I am offering a more radical and controversial combination for changing current practice.

\section{b) Non significance for conventional and Bayesian analysis, with the same data and shift of priors}

Suppose I am more skeptical about the claim before the Ulrich study and think there is only a $20 \%$ chance of it being true (i.e. I set the priors at 0.2 and 0.8 ). Then

$p(H 1 / E 1)=(0.9 \times 0.2) /((0.9 \times 0.2)+(0.3 \times 0.8))=$ 0.42 with $p(-H 1 / E 1)=0.58$.

Now, under the same evidential data, my post study belief in Ulrichs claim has strengthened from 0.2 up to 0.42 . However belief in the alternative hypothesis while weakening from $80 \%$ to $58 \%$ is still stronger and so we remain skeptical and do not accept Ulrich's claim. The Bayesian approach can easily be integrated into decisional frameworks. For example, using the Delphi technique [22], initial disagreement could be reduced so that a professional consensus view could be achieved. This could result in some form of knowledge base for the discipline for those issues which were more controversial. The good news as stated earlier is that convergence occurs fairly quickly, irrespective of initial prior values. 